\title{
Loop Mirror Multi-wavelength Brillouin Fiber Laser Utilizing Semiconductor Optical Amplifier and Fiber Bragg Grating
}

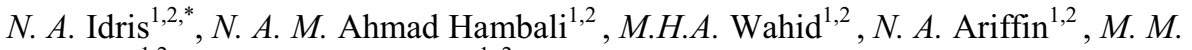 \\ Shahimin $^{1,2}$, and M.A.M. Azidin ${ }^{1,2}$ \\ ${ }^{1}$ School of Microelectronic Engineering, Universiti Malaysia Perlis (UniMAP), Pauh Putra, 02600 \\ Arau, Perlis, Malaysia \\ ${ }^{2}$ Semiconductor Photonics \& Integrated Lightwave Systems (SPILS), School of Microelectronic \\ Engineering, Universiti Malaysia Perlis, Pauh Putra, 02600 Arau, Perlis, Malaysia
}

\begin{abstract}
In this paper, the development of loop mirror multi-wavelength Brillouin fiber laser utilizing semiconductor optical amplifier and fiber Bragg grating is successfully demonstrated. A multi-wavelength BFL structure employs a single mode fiber that acts as the gain medium, while the fiber Bragg grating act to reflect the signals and semiconductor optical amplifier is used to amplify the signal are employed to produce multiple channels. The implementation of difference parameter for semiconductor optical amplifier driven current, Brillouin pump power, Brillouin pump wavelength and length of single mode fiber, plays an important role in producing the multiple channels. The highest number of channels which is 15 achieved once $9 \mathrm{~km}$ of SMF length, semiconductor optical amplifier current at $800 \mathrm{~mA}$ and low Brillouin pump power at $-12 \mathrm{dBm}$ are utilized in the laser structure. Furthermore, the implementation of semiconductor optical amplifier and reflectivity of fiber Bragg grating in Brillouin fiber laser structure gives a better performance in producing the multiple channels.
\end{abstract}

\section{Introduction}

Throughout the years, multi-wavelength fiber laser has received much attention due to their numerous works with potential applications in optical fiber sensors [1], wavelength division multiplexing (WDM) systems [2], spectroscopy [3] and optical component testing [4].Various amplification methods have been proposed previously in producing the multiwavelength output, such as semiconductor optical amplifier (SOA), Erbium-doped fiber amplifier (EDFA), and Raman amplifier [4].Typically, erbium-doped fiber amplifier (EDFA) is a favourable candidate in generating multiple channels which is has the capability of providing large gain, high saturation power and also low noise figure [5]. However, due to homogenous broadening mechanism from the erbium doped fiber which is

*Corresponding author: azuramalini@unimap.edu.my 
caused by the competition between the laser modes and leads to fluctuating of output channels from laser system [5].

Thus, the low number of channels and unstable channels are produced. To overcome this problem, the inhomogeneous broadening characteristic that offered by SOA is the alternative way in producing a large number of channels [6]. Besides that, it minimizes the competition of the laser mode and has the ability to produce a stable multiple channels. The SOA has remarkable advantages, mainly due to compactness in terms of size, light weight, lower power consumption and also mass producible [6].

The Brillouin fiber laser (BFL) is one type of fiber laser that experienced a nonlinear effect known as Stimulated Brillouin Scattering (SBS). This nonlinear process involves the interaction between the intense pump light that being injected by the light source and acoustic wave in the optical fiber [7]. Through this process, a unique result is produced where the generated output signal propagate in backward direction and possess a downshifted frequency from the injected signal [7]. Based on the previous works, the generation of Brillouin semiconductor fiber laser associated with SOA and EDFA have been conducted. From this paper, the implementation of the EDFA as signal amplifier in producing multi-wavelength is restricted due to homogenous broadening effect. The improvement has been made by the use of SOA which able to produce a multi-wavelength in any wavelength of the outpurt power. However, only small peak of channels and low OSNR values are produced as output.

Thus, the loop mirror multi-wavelength Brillouin fiber laser (MWBFL) utilizing SOA and reflectivity of FBG has been studied to make an improvement in term or number of channels which is much highest that previous studies in [8]. In this paper, the performance of the BFL utilizing SOA and FBG was demonstrated. Implementation of SOA and FBG are resulted the better performance in producing the multiple channels. The FBG comprises of a special characteristic which is refractive index in the fiber core which leads the light ray to reflect back in generating a multiple channels [9]. Evidently, the highest number of channels is produced by proposed MWBFL structure utilizing SOA and FBG. At $-12 \mathrm{dBm}$ of Brillouin pump (BP) power and $1550 \mathrm{~nm}$ BP wavelength, 15 channels are produced. The variation of SOA driven current, BP wavelength and length of SMF affect the performance and generation of the channels.

\section{Simulation and illustration of operating principle}

The simulation design and structure illustration of loop mirror MWBFL utilizing SOA and FBG are shown in Fig 1 and Fig 2, respectively. Based on this structure, the external continuous wave $(\mathrm{CW})$ laser plays the function as a Brillouin pump (BP) signal in the range of $-12 \mathrm{dBm}$ to $6 \mathrm{dBm}$. The SMF functions as Brillouin gain medium in the range of $7 \mathrm{~km}$ to $14 \mathrm{~km}$ length. In producing the multiple channels, reflection of FBG is the vital element in BFL structure. The FBG's has the characteristic of having central wavelength of $1550 \mathrm{~nm}$. The $3-\mathrm{dBm}$ of bidirectional optical coupler and optical circulator are used to extract the output and to prevent the signal from propagating in random direction [9], respectively. The BP power and BS signal undergoes signal amplification by SOA with driven current of $350 \mathrm{~mA}$ to $800 \mathrm{~mA}$. The optical spectrum analyzer (OSA) is external elements that serve the purpose to measurement and observation output results. 


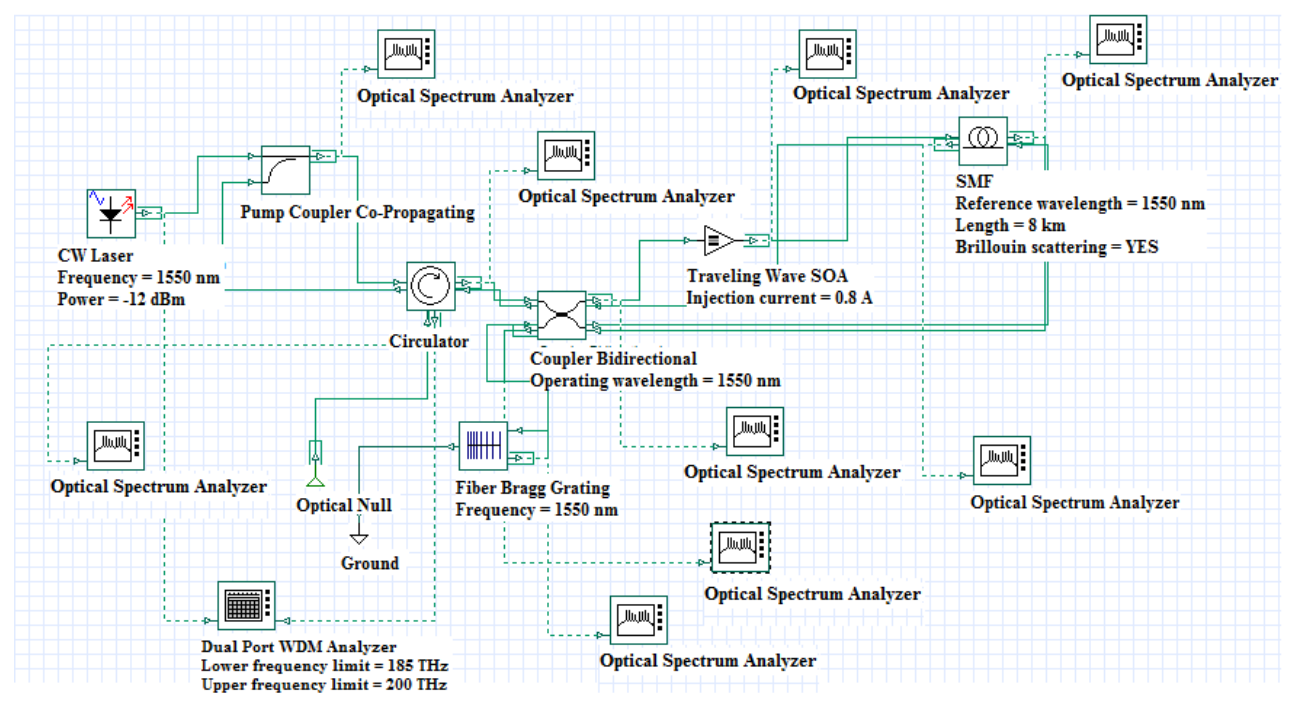

Fig. 1.The simulation design of loop mirror MWBFL utilizing SOA and FBG.

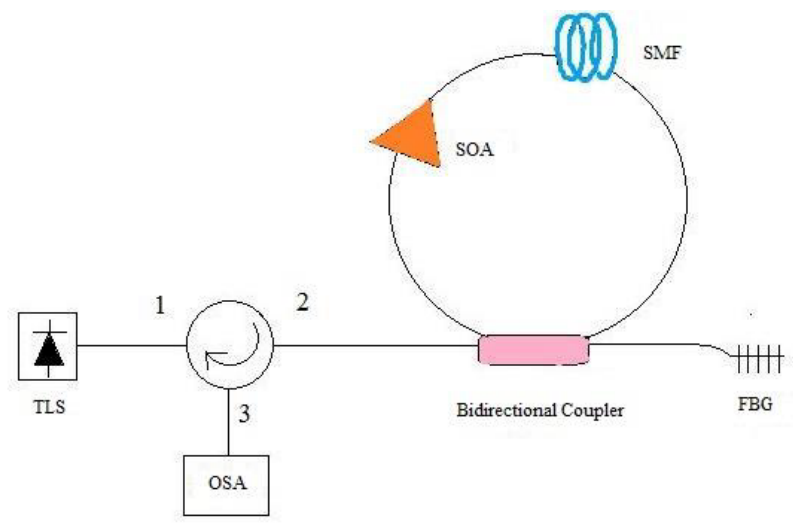

Fig. 2. The structure illustration of loop mirror MWBFL utilizing SOA and FBG.

The operation started by the injection of the BP signal from the external CW laser into the optical circulator where the signal is travelled from port 1 to port 2. Next, the BP signal passed through the bidirectional optical coupler and propagated into SOA. The amplified and stronger BP signals were produced in the SOA as it has a special feature to boost up the intensity of the optical signal [9]. Then, the BP signal is propagated into the SMF and experiences the SBS effect. After the Brillouin threshold condition in SMF is achieved, the BS signal known as channels is produced. The BP signal is then propagated to the FBG and reflected back in the loop mirror structure. Each of the generate BS signal act as a new pump to generate the next order of BS signals generation. After that the BS signal (channels) travelled from the bidirectional optical coupler and optical circulator (port 2 to port 3) and can be observed and measured through the OSA with bandwidth resolution of $0.01 \mathrm{~nm}$. 


\section{Experimental result and discussion}

Figure 3 shows the evolution of the number of channel plotted against the difference values of BP power. The BP power is injected into MWBFL structure in the range of $-12 \mathrm{dBm}$ to 6 $\mathrm{dBm}$ with $1550 \mathrm{~nm}$ of $\mathrm{BP}$ wavelength. The implementation of different SOA driven currents produced a different number of channels. At $800 \mathrm{~mA}$ and $3 \mathrm{dBm}$ of $\mathrm{BP}$ power, 15 channels are generated, followed by $700 \mathrm{~mA}, 650 \mathrm{~mA}$ and $350 \mathrm{~mA}$ which is produced about 6,4 and 2 channels, respectively. This condition shows that the increment of the SOA driven current arise the number of channels produced due to sufficient energy. As the BP power increases to $6 \mathrm{dBm}$, the number of channels shows a slightly decrease due to several factor such as temperature, noise and the gain that shared among the channels to grow their peak power. This factor affected the performance in generating a number of channels.

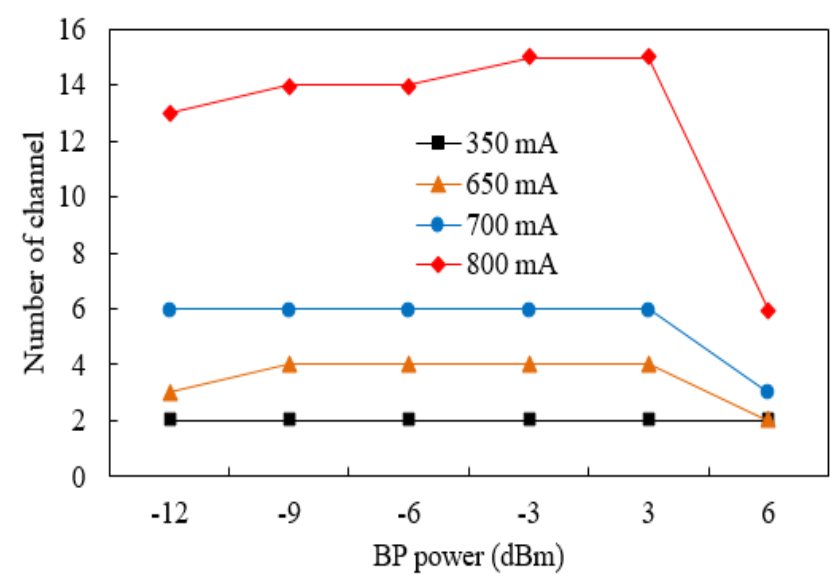

Fig. 3.The number of channels versus BP power at BP wavelength of $1550 \mathrm{~nm}$ with SOA driven current of $800 \mathrm{~mA}$.

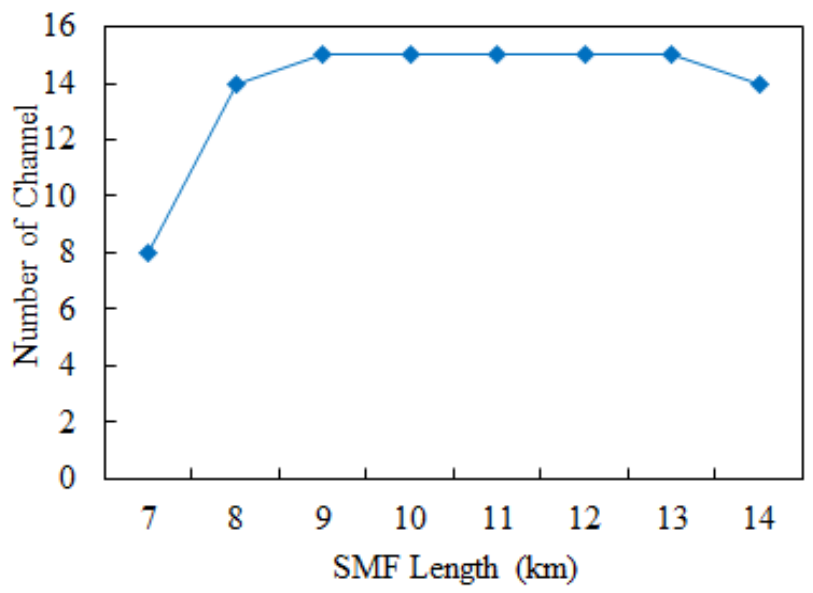

Fig. 4. The number of channels versus SMF length at $-12 \mathrm{dBm}$ of injected power and $800 \mathrm{~mA}$ of SOA driven current. 
Next, the relationship between the numbers of channels versus SMF length and output spectra is plotted in Fig 4. The length of SMF is varied from $7 \mathrm{~km}$ to $14 \mathrm{~km}$ with the SOA driven current is fixed at $800 \mathrm{~mA}$ and BP power is injected at $-12 \mathrm{dBm}$. From plotted graph, the MWBFL experienced an increasing number of channels as the length of the SMF is increases. When, the SMF length is increases to $14 \mathrm{~km}$, it shown the decrement of number of channel. This factor is due to the attenuation in the fiber such as light absorption, scattering and radiation loss. Furthermore, the output spectra for different length of SMF with BP wavelength of $1550 \mathrm{~nm}$ and SOA driven current of $800 \mathrm{~mA}$ are shown in Figure 5.

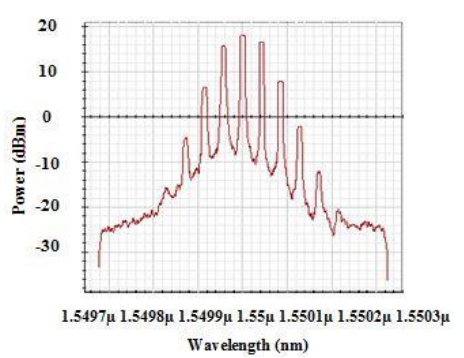

(a)

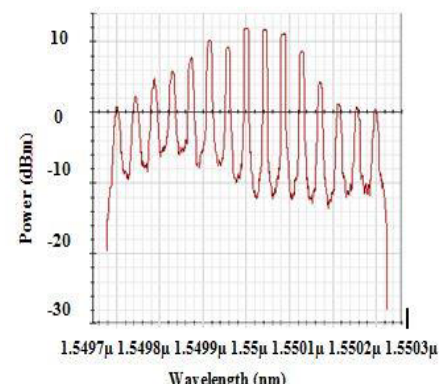

(d)

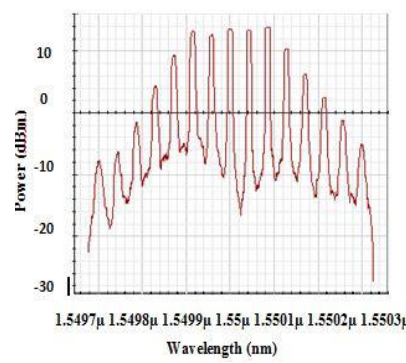

(b)

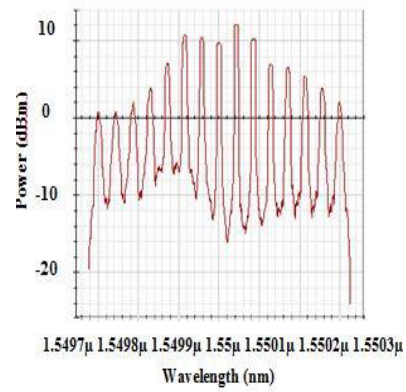

(e)

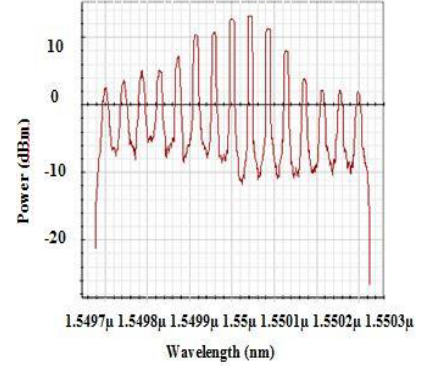

(c)

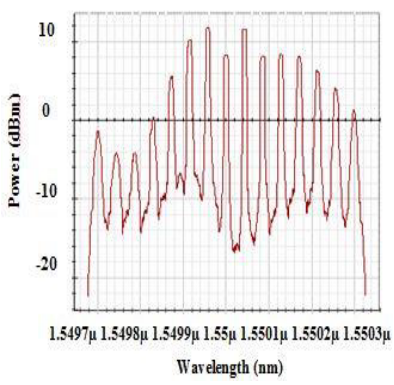

(f)

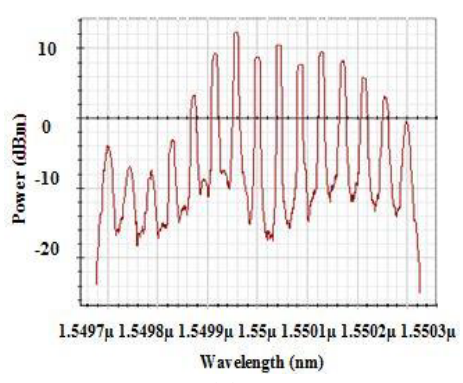

(g)

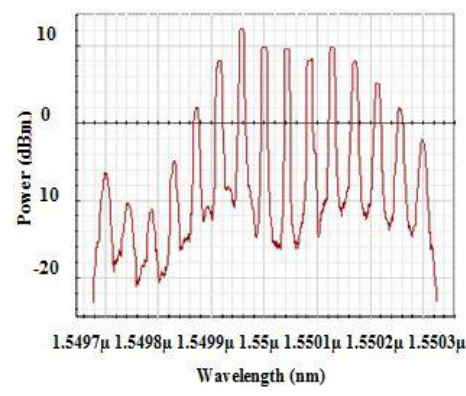

(h)

Fig. 5.The output spectra for different length of SMF with BP wavelength of $1550 \mathrm{~nm}$ and SOA driven current of $800 \mathrm{~mA}$. (a) $7 \mathrm{~km}$ (b) $8 \mathrm{~km}$ (c) $9 \mathrm{~km}$ (d) $10 \mathrm{~km}$ (e) $11 \mathrm{~km}$ (f) $12 \mathrm{~km} \mathrm{(g)} 13 \mathrm{~km}$ and (h) $14 \mathrm{~km}$.

Further analysis of the generation multi-wavelength BFL utilizing SOA and FBG is continued as shown in Fig 6. The BP wavelength is varied from $1530 \mathrm{~nm}$ to $1565 \mathrm{~nm}$ which enters the window transmission of conventional band (C-band). Meanwhile, the BP power and SOA driven current are fixed at $-12 \mathrm{dBm}$ and $800 \mathrm{~mA}$, respectively. Based on Fig 6, 14 number of channels are produced in the range of $1530 \mathrm{~nm}$ to $1555 \mathrm{~nm}$ BP wavelength. The $\mathrm{BP}$ wavelength experienced a decrease in the number of channels at $1560 \mathrm{~nm}$ to $1565 \mathrm{~nm}$. 


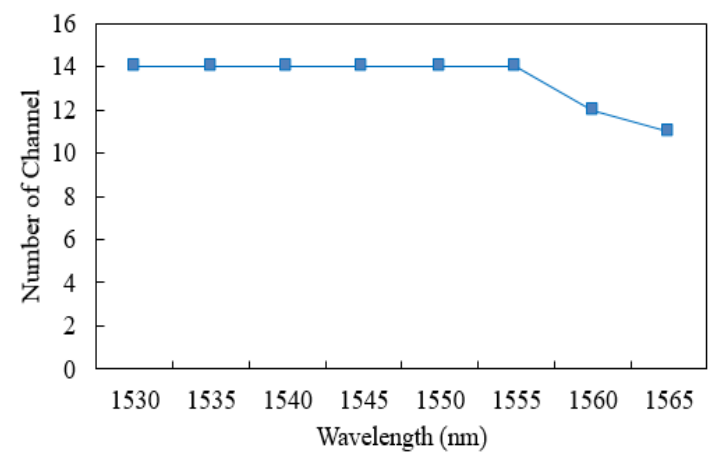

Fig. 6. The number of channels versus wavelength with BP power of $-12 \mathrm{dBm}, 800 \mathrm{~mA}$ of SOA driven current, $8 \mathrm{~km}$ of SMF and BP wavelength at $1550 \mathrm{~nm}$.

\section{Conclusion}

As presented in this paper, the development of the loop mirror MWBFL utilizing SOA and FBG is successfully demonstrated in the C-and region which in the range of $1530 \mathrm{~nm}$ to $1565 \mathrm{~nm}$. The implementation of SOA and FBG plays the important role in producing multiple channels. At BP power of $-12 \mathrm{dBm}$, SMF length of $9 \mathrm{~km}$ and SOA driven current of $800 \mathrm{~mA}, 15$ number of channel are obtained. Besides that, 14 channels are produced due to the range of BP wavelength of $1530 \mathrm{~nm}$ until $1555 \mathrm{~nm}$. Furthermore, it can be concluded that the performance of the loop mirror MWBFL is dependent on the characteristics of SOA and FBG to produce multiple channels and has great potential in DWDM system.

This work was fully supported by the Ministry of Higher Education, Malaysia under research grant \#FRGS/9003-00532\#

\section{References}

1. N.S. Shahabuddin, S.W. Harun H.Ahmad, J. Optoelectron. Adv. Mater., 3, 1 (2009)

2. H. Ahmad, N.A. Hassan, M.H. Jemangin, S.W. Harun, M.Z. Zulkifli, J. Quantum Electron.,7, 602 (2011)

3. K.K. Qureshi, H.Y. Tam, Opt. Laser Technol., 44, 1646 (2012)

4. S.F. Norizan, M.Z. Zulkifli ,Z.A. Ghani, S.W. Harun, H. Ahmad, Fiber Optics, 21, 1 (2011)

5. A.H. Sulaiman, S. Shahi, S.W. Harun, H. Ahmad, Fiber Optics, 19, 5 (2009)

6. H.C. Ooi, A.H. Sulaiman, K. Thambiratnam, M.Z. Zulkifli, S.W. Harun H. Ahmad, Microw. Opt. Technol., 50, 20 (2008)

7. C. Wei, T.X. Bo, M.Z. Hu, X. Yang, Chin. Phys., 23, 12 (2014)

8. A. Hayder, R.C. Lawrence, IEEE, 19, 398 (2006) 